\title{
Practical staining method of well-unstained haematological specimens
}

\section{Abstract}

In hematology, May-Grunwald-Giemsa (MGG) staining is used to evaluate bone marrow aspirate as well as blood smear. This is an easy method in order to avoid the damage of the cells; good qualities of the stain and its directives, as well as the duration of the procedure are very important. The smears need to be dried quickly after the procedure. For a successful smear needs to be thin and while drying it up, hot blowing can be used. In case bone aspirate or blood smear are not well stained by MGG, the aspiration has to be re-executed or the biopsy results should be awaited. That procedure takes approximately 15 days that is needed to get the biopsy result and a long time that leads to material and motivational loss. In our clinic, we use a practical stain method for pre-diagnosis in such cases. Two cases are presented in this study inform you about that method that yielded good results on a first trial.

Keywords: may-grunwald-giemsa, bone marrow aspirate, pale staining, lamella, biopsy
Volume 4 Issue 5 - 2017

\section{Osman Yokuș,' Habip Gedik, ${ }^{2}$ Ezgi Ceren} Sahin ${ }^{3}$

'Department of Hematology, Ministry of Health İstanbul Training and Research Hospital,Turkey

2Department of Infectious Diseases and Clinical Microbiology, Ministry of Health Bakırköy Sadi Konuk Training and Research Hospital,Turkey

${ }^{3}$ Istanbul School of Medicine, Istanbul University, Turkey

Correspondence: Habip Gedik, Department of Infectious Diseases and Clinical Microbiology, Ministry of Health Bakırköy Sadi Konuk Training and Research Hospital,Turkey, Tel +90 212 31455 55, Fax +902122217800

Email habipgedik@yahoo.com

\section{Case reports}

\section{Case I}

28 years old male patient diagnosed with Acute Myeloid Leukemia had $3+7$ chemotherapy. On the $28^{\text {th }}$ day of the treatment after the peripheral blood smear results improved; bone marrow aspirate was pale, although all the cells were stained (Figure 1). The second stain was performed and the results were perfect. Less than $5 \%$ of blasts were seen supporting the idea of remission (Figure 2).

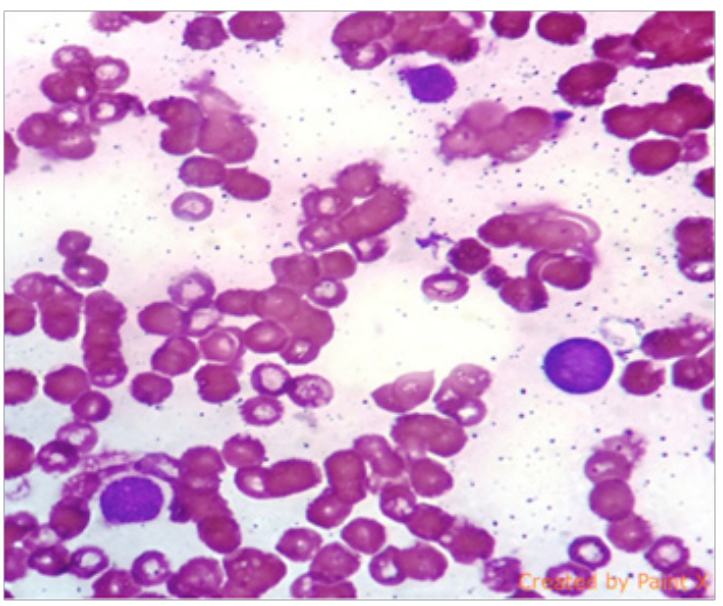

Figure I Control of AML case after remission induction therapy. Bone marrow sample was bad stained and cells do not look good.

\section{Case 2}

We were asked for consultation of 38years old pancytopenia patient from department of internal medicine. On the first try of the staining, we could not get the result due to pale staining (Figure 3). The second staining was performed and results were satisfied (Figure 4).

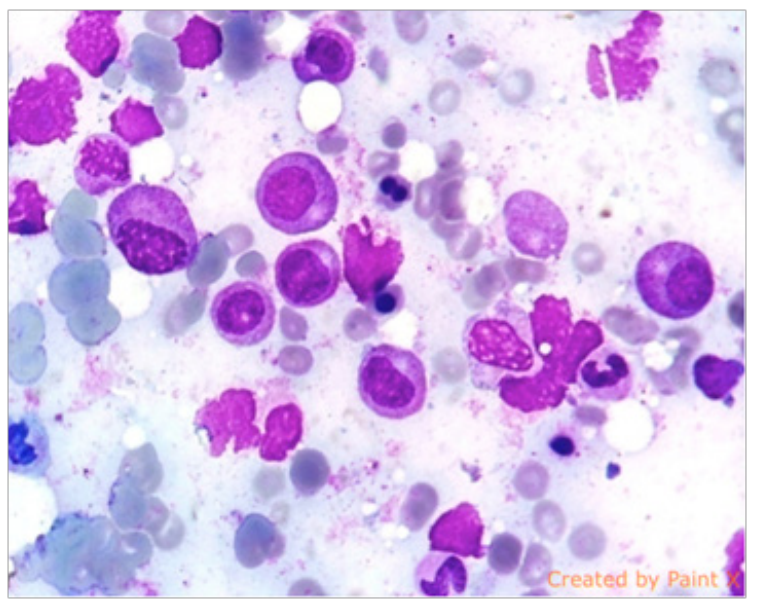

Figure 2 After the second staining of the same sample of AML case, the cells of Figure 3 look better.

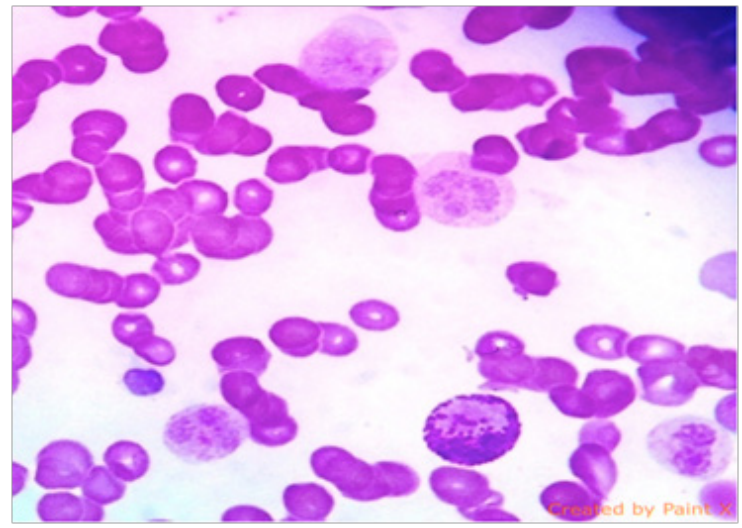

Figure 3 The bone marrow aspirate of case with pancytopenia. The cells are flu and the details are not obvious. 


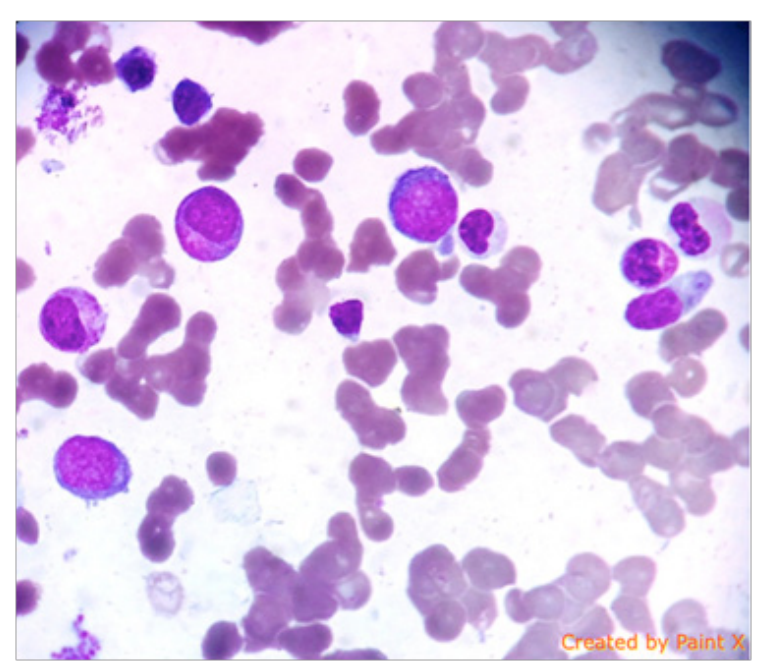

Figure 4 The cells are seen better after the second staining of Figure 3.

\section{Discussion}

Although there is a little difference among medical centers, the summary of the techniques of MGG staining is like that: Bone marrow aspirate or blood smear which is dried in the ambient temperature is covered pouring May-Grunwald (5-7drops) and then it is waiting two minutes. Then it is poured away (without washed by tap water) and prepared Giemsa staining (5-7drops) with distilled water is poured onto lamella waiting for 7 minutes. Then lamella is washed with tap water that is quickly dried and examined under the microscope.
With this technique, if the lamella is not stained well, or the sample is pale, our experience is that those samples could be stained well executing shorter staining time with less amount of dye. We pour over the samples with May-Grunwald for $1.5 \mathrm{~min}$ and then pour cover them with Giemsa for 4min. After that, we wash the lamella in the same way under tape water, dry up and then examine. As seen in four pictures, the samples that could not be examined are well stained for examination.

\section{Conclusion}

As a result, when an insufficiently stained sample is dyed again with the same stain procedure in shorter period, it is morphologically evaluated better. This method prevents a great waste of time and suffering of patient with a re-biopsy. ${ }^{1}$ In practical hematology applications, this procedure will be useful and provide exact results. As we want to share this practical method with our colleagues, although there may be some with different experiences.

\section{Acknowledgements}

None.

\section{Conflict of interest}

The author declares no conflict of interest.

\section{References}

1. Ibrahim Ramzy. Clinical Cytopathology and Aspiration Biopsy. 2nd ed. New York, USA: McGraw-Hill Education-Europe; 2001. p. 587-598. 